\title{
Solid State Coherent Transient Measurements Using Hard Optical Pulses
}

\author{
G. J. Pryde, M. J. Sellars, and N. B. Manson \\ Laser Physics Centre, Research School of Physical Sciences and Engineering, The Australian National University, \\ Canberra, ACT, 0200, Australia
}

(Received 23 August 1999)

\begin{abstract}
An isolated and spectrally narrow absorptive feature is prepared via a novel spectral hole burning process in an inhomogeneously broadened optical transition in $\mathrm{Eu}^{3+}: \mathrm{Y}_{2} \mathrm{SiO}_{5}$. With the narrow feature it is shown that it is feasible to apply complex optical pulse sequences analogous to rf pulse sequences used extensively in NMR.
\end{abstract}

PACS numbers: $42.50 . \mathrm{Md}, 42.50 . \mathrm{Hz}, 78.47 .+\mathrm{p}$

In this paper we introduce a new experimental technique whereby multiple pulse coherent transient techniques frequently used in the field of NMR can be successfully applied to an optical transition in a solid. The coherent transients associated with NMR multiple pulse experiments in the radio frequency region of the spectrum have enjoyed enormous success $[1,2]$ providing a wealth of structural and dynamical information. Analogous experiments at visible wavelengths should be just as informative, but until now it has not been possible to apply the necessary "hard" optical pulses. In this work it is shown that for certain kinds of materials there is a novel solution to this problem and the technique is demonstrated for a rare-earth doped solid.

Multiple pulse techniques involve accurately manipulating the quantum states of ensembles in order to isolate interactions of interest [2]. This requires the use of fully hard pulses where the optical field is sufficiently intense that all the ions excited experience the same generalized Rabi frequency. This in turn entails working with Rabi frequencies much larger than the transition linewidths. This has not been achieved in solids. In the case of $f^{n}-f^{n}$ rare-earth ion transitions the optical inhomogeneous linewidths are several GHz and the oscillator strengths are low. The required $\mathrm{GHz}$ Rabi frequencies cannot be reached with $\mathrm{cw}$ lasers, which must be used so as to have control over delay, duration, amplitude, and phase of the applied pulses. Thus, although rare-earth transitions are particularly amenable to coherent transient studies, measurements are necessarily restricted to the simpler pulse techniques, such as two pulse photon echoes, where hard pulses are not a requirement [3]. Only one multiple pulse technique has been attempted in rare-earth doped solids. Erickson attempted to use a Carr-Purcell $(\mathrm{CP})$ pulse sequence to measure the optical dephasing rate in $\mathrm{LaF}_{3}: \mathrm{Pr}^{3+}$, but concluded that the soft pulses resulted in the creation of population gratings across the inhomogeneous line [4]. Excitation of these gratings produced stimulated echoes, swamping the desired signals.

In the present work before applying the multiple optical pulse sequence, we implement a vital additional procedure, which changes the essential character of the inhomogeneous line. Rather than operating with the entire inhomogeneous linewidth we use spectral hole burning to prepare a narrow positive (absorptive) spectral feature. The subsequent multiple pulse sequence excites only the specially selected subgroup of centers associated with this narrow feature. The feature is spectrally narrow enough for the exciting pulses now to be considered hard. Hence, the narrow spectral feature entirely changes the viability of multiple pulse techniques. In the present paper the successful application of the approach is demonstrated for a CP Meiboom-Gill (CPMG) sequence [5], one of the basic multiple pulse sequences in NMR.

The technique is applicable to hole burning materials and for this demonstration we used a sample of $\mathrm{Eu}^{3+}$ : $\mathrm{Y}_{2} \mathrm{SiO}_{5}\left(0.5\right.$ at $\left.\% \mathrm{Eu}^{3+}\right)$ cooled to $1.6 \mathrm{~K}$. There are two $\mathrm{Eu}^{3+}$ sites in this crystal, and the present measurements were made on the ${ }^{7} F_{0^{-}}{ }^{5} D_{0}$ transition at $579.879 \mathrm{~nm}$. The inhomogeneous broadening due to crystal strain is $11 \mathrm{GHz}$ and the peak absorption is $40 \%$. The lifetime is $1.9 \mathrm{~ms}$ [6] and at low concentrations $(0.1 \%)$ the reported dephasing time in zero magnetic field is $1.5 \mathrm{~ms}$ [6]. The ground and excited states are orbital singlets, but each has three hyperfine levels associated with the ${ }^{151} \mathrm{Eu}$ and ${ }^{153}$ Eu's nuclear spin of $5 / 2$. The spectrum of each isotope is expected to be dominated by three spin-allowed transitions of near equal oscillator strength. The other six transitions are weakly allowed and give rise to the hole burning with a lifetime of hours [7].

The measurements were made using the setup of Ref. [8]. A highly stabilized tunable dye laser was developed, and the relevant stability has been established to be $1 \mathrm{kHz}$ over time scales up to $1 \mathrm{~s}$. The light pulses were gated by two acousto-optic modulators (AOMs) in series and these also control the relative phase of the pulses. To enable phase sensitive detection, a Mach-Zehnder interferometer was employed. The net frequency difference between the local oscillator and signal beam, introduced by the AOMs, was set to $10 \mathrm{MHz}$. The heterodyne signal at this frequency was detected using a photodiode. The beat signal was then combined in phase and in quadrature with the $10 \mathrm{MHz}$ rf source to detect the two orthogonal phases of the signal. To reduce the effective range of intensities across the sample, an aperture was placed in the reference arm at the point equivalent to the position of 
sample in the test beam. As the reference beam and the test beam were mode matched along their length, this was equivalent to masking the sample, but considerably more convenient. As evidence of its effectiveness, insertion of the aperture was found to improve the visibility of the nutations observed in rotary echo measurements [9]. The nutation frequency was used to set pulse areas in subsequent experiments. The signals were obtained with sufficient signal to noise to make averaging unnecessary.

The novel aspect of this work, the preparation of the narrow spectral feature, is achieved in two steps; one step selects the frequency and the other selects the oscillator strength. To select the frequency a temporally shaped pulse is applied which has zero amplitude at the laser frequency and appreciable amplitude at adjacent frequencies. The pulse consists of two rectangular pulses of equal areas but opposite phases, one short pulse and the other long (Fig. 1). The pulse area of the individual pulses was of the order of $\pi / 4$. We demonstrate the process using a short intense pulse of $1 \mu$ s to excite ions over a $1 \mathrm{MHz}$ band with a weaker long pulse of $40 \mu$ s returning a much narrower $(\sim 25 \mathrm{kHz})$ group of ions at the central frequency to the ground state. After optical relaxation a proportion of the absorption over the wide bandwidth is lost through spectral hole burning, whereas the absorption at the central frequency is left unchanged. The result is a narrow absorption feature at the center of a broad hole. The depth of the hole can then be enhanced by repeating the double pulse sequence and is repeated 130 times at intervals of $4 \mathrm{~ms}$, a time considerably longer than the optical lifetime. The processes are modeled in Fig. 1. The spectrum was calculated using optical Bloch equations to determine the excited state population immediately after the application of the pulses. To match the observed hole depth, it is assumed that after each application of the pulse pair, $6 \%$ of the excited ions do not return to their initial ground state.

The second step of the preparation is the selection of ions on the basis of the Rabi frequency. The variation in Rabi frequency will arise from the variation in the oscillator strength and in particular the presence of the six weaker transitions. To reduce the distribution of Rabi frequencies we apply a series of five pulses, exactly $2 \pi$ in area and $6.2 \mu \mathrm{s}$ long, separated by $4 \mathrm{~ms}$ time intervals. The $2 \pi$ pulses lead to preferential hole burning of ions experiencing pulse areas other than $2 \pi$ and will reduce the contribution of the weaker transitions. The process generates an absorption feature that has both a well-defined frequency and oscillator strength. Spectral features produced by the above procedure were observed up to $30 \mathrm{sec}$ after their creation, with no measurable change in their characteristics.

The narrow spectral feature is investigated using further coherent transients. The three pulse sequence given in Fig. 2(i) consists of a $\pi / 2$ pulse followed, after a delay, by a $\pi$ pulse with a $90^{\circ}$ phase shift and after a longer delay by a second $\pi$ pulse in phase with the first $\pi$ pulse. The transient response presented in Figs. 2(ii) and 2(iii) was obtained with single applications of this three pulse sequence. Averaging was unnecessary. Consistent with expectations, all the subsequent signals are in phase and are shown in the experimental trace in Fig. 2(ii). Correspondingly, there are only weak signals in the quadrature phase, and for brevity this phase is not shown.

There are long free induction decay signals after each of the applied pulses. Long responses confirm the presence of a narrow spectral feature. Also the relative sizes of these signals are of interest. The signal is larger for first pulse than for the second pulse of twice the duration, agreeing with expectations that coherence is maximized for resonant $\pi / 2$ pulses but is zero for $\pi$ pulses. Coherence would also be established if off-resonant excitation were involved. Hence, the large signal for the first pulse $(A)$ and small signals for the second pulse ( $B$ and $E$ later) confirm that these pulses are very close to the ideal hard $\pi / 2$ and $\pi$ pulses, respectively. Fast free induction decay present due to the excitation of any broad band feature is

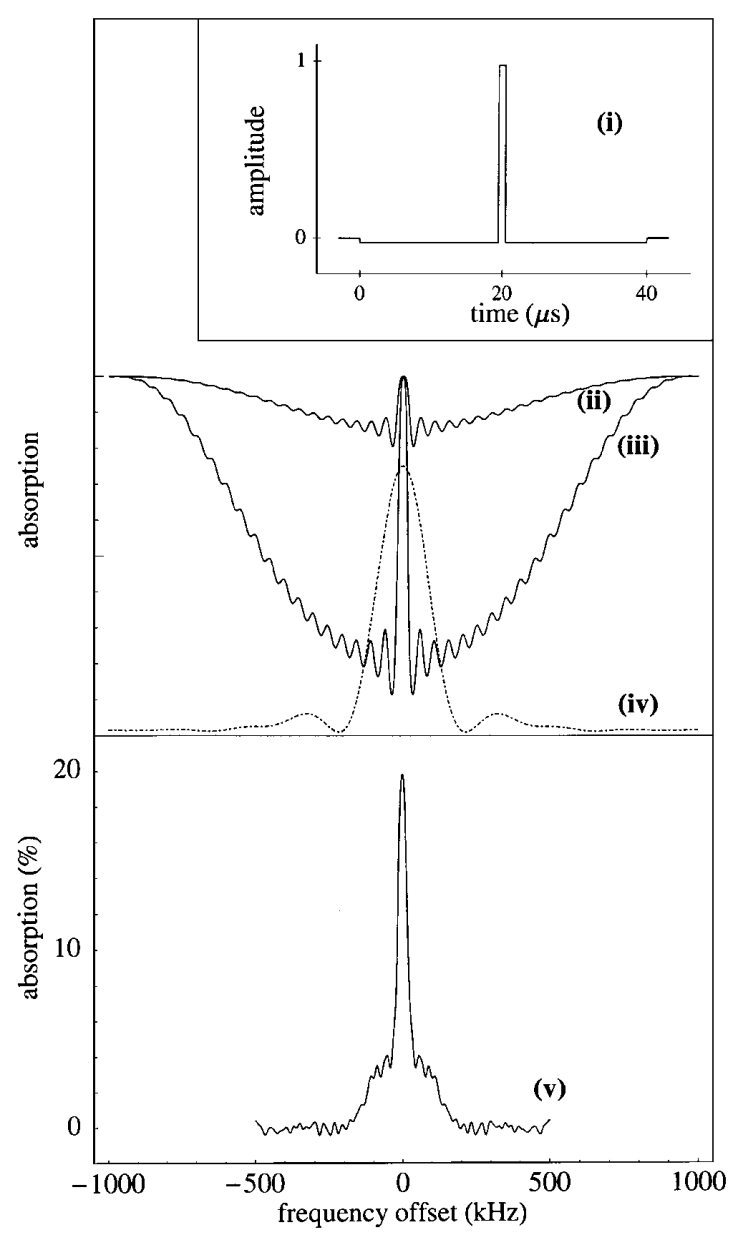

FIG. 1. Calculated and experimental hole burning spectra of the narrow absorptive feature. Inset (i) shows the pulse pair used to burn the spectral hole. The calculated hole shape (ii) following 13 repetitions of this pulse pair and (iii) after 130 repetitions. (iv) The region of the spectrum selected by the pulses in the CPMG experiment. (v) Fourier transform of the echo obtained in the experiment. 


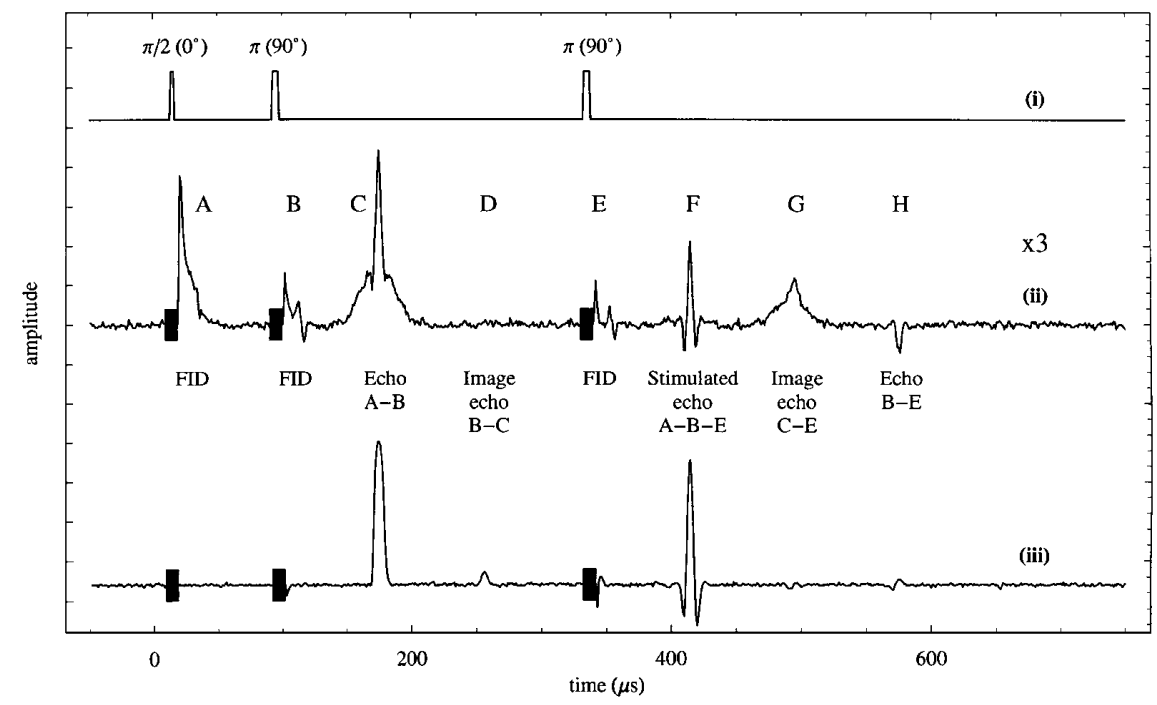

FIG. 2. Experimental results of the prototype Carr-Purcell experiment performed on the narrow feature and on the unmodified inhomogeneously broadened line. The letters $A$ to $H$ represent notable features. (i) The pulse sequence applied, with the phase shift shown in brackets. (ii) $0^{\circ}$ phase sensitive detected signal from the sample containing the narrow spectral feature. (iii) gives the $0^{\circ}$ phase sensitive detected signals from the unmodified inhomogeneous line.

lost in the detection dead period (indicated by solid rectangles in Fig. 2) following the saturation of the detector by the applied light pulses. However, the echoes are well clear of these periods, and it can be seen that the signals associated with the first photon echo $(C)$ have a short and a long component. The Fourier transform yields a narrow spectral feature superimposed on a weak broad background, the latter component being related to the remnant of the unburned inhomogeneous line. This spectrum is shown in Fig. 1(iv) where it can be seen to be in good correspondence with the predicted feature. The ratio of the peak of the narrow absorptive feature to the unburned background is $5: 1$.

The third pulse in the sequence was introduced to assess whether the narrow feature would be suitable for more complex transient studies. There are three extra echoes introduced by the third pulse $(E)$. The image echo $(G)$ is associated with rephasing for the second time the coherence initially generated by the $\pi / 2$ pulse $(A)$. As with the first rephasing, the photon echo $(C)$, it has a short and a long component. It is significant that there is no equivalent long duration stimulated echo $(F)$. This is because there is no grating formed within the narrow spectral feature, a consequence of having a single Rabi frequency over the narrow line (i.e., hard pulses). This is the most significant conclusion of the preliminary investigation, as it is precisely what is required in multiple pulse sequences, where it is necessary to measure the magnitude of the image echo signal under conditions where the echoes $F$ and $G$ coincide. This is trivial as a consequence of there being no broad stimulated echo.

As the short duration echo signals are associated with the weak unburned background they can be related to the responses obtained when applying the same pulse sequence of the unmodified inhomogeneously broadened line, shown in Fig. 2(iii). There is a marked contrast between the signals obtained for the modified and unmodified line. For the unmodified line there are, of course, no long duration signals, and there is no narrow feature. All responses are of short duration, including the free induction decays, which are completed during the detection dead period. Also the image echo is significantly smaller than the stimulated echo. The presence of the very large stimulated echo makes the unaltered inhomogeneous line totally inappropriate for complex pulse sequences confirming Erickson's conclusions [4]. In the case of the prepared sample the short duration echoes are attenuated by a factor of 6 , consistent with the reduction in the broad background (5:1). The short duration features are superimposed on the longer duration signals but are easily distinguished. Thus, the presence of the broad background does not prevent us from monitoring the coherent transient response associated with the sharp spectral line.

As a demonstration of a multiple pulse experiment we excited the sample with a CPMG pulse sequence. The sequence is commonly used in the field of NMR to measure the dephasing time of transitions. It has the advantage over a simple two pulse echo that the decay time can be obtained in a single shot [5]. It is also used to study the time scale of the perturbations that give rise to the dephasing. The sequence is related to the three pulse sequence investigated above. It consists of a $\pi / 2$ pulse followed by a train of $\pi$ pulses all phase shifted by $90^{\circ}$ with respect to the first pulse. The separation between the $\pi / 2$ and the first $\pi$ pulse is half that between the successive $\pi$ pulses. Between each pair of $\pi$ pulses the coherence established by the first pulse is rephased to produce the photon echo of interest. The CPMG was chosen in preference to the straight Carr-Purcell sequence because cumulative errors due to incorrectly set pulse areas do not arise [1]. Figure 3 


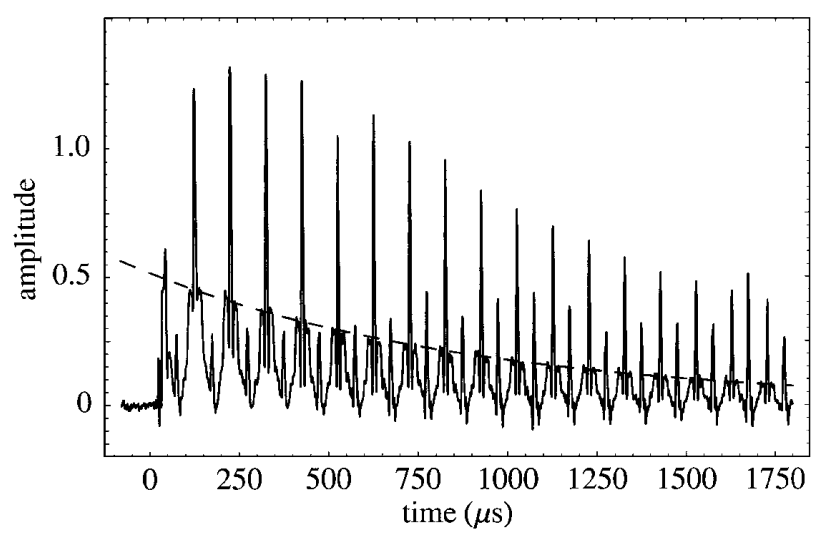

FIG. 3. The $0^{\circ}$ phase sensitive detected coherent transient signal resulting from the application of a Carr-Purcell MeiboomGill pulse sequence using a sample of $\mathrm{Eu}^{3+}: \mathrm{Y}_{2} \mathrm{SiO}_{5}$ that had been previously prepared to contain a narrow spectral feature $25 \mathrm{kHz}$ wide.

shows the result of applying a single CPMG sequence after preparing the narrow feature. The dephasing time is given by the decay of the long duration echo features and have a value of $T_{2}=900 \mu \mathrm{s}$.

The dephasing rate was also measured using the conventional two pulse photon echoes without any preparation to the inhomogeneous line. The measurement was made under the identical conditions as for the CPMG experiment with pulses of the same duration and intensity. The value of $T_{2}$ obtained using a series of such measurements gave $260 \mu \mathrm{s}$. The shorter dephasing time arises from time varying fields produced by optical excitation of neighboring ions perturbing the ion of interest (instantaneous spectral diffusion) $[6,10]$. The more ions excited the larger the effect, which hence increases with laser intensity, concentration, and excited bandwidth. The use of the narrow spectral feature as expected reduced the effects of instantaneous spectral diffusion by reducing the density of ions excited. It is not completely eliminated, as there is still a contribution from exciting ions in the unburned background as well as ions in the line itself. Longer dephasing times have been established by Equall et al. [6] using an alternative approach where they minimized the excitation density by reducing concentration and laser intensity. In this way, for a sample of $0.1 \%$ they obtained a dephasing of $1.5 \mathrm{~ms}$. In the present work although instantaneous spectral diffusion has not been eliminated, the new techniques provide additional flexibility for the study of such effects. For example, it would be straightforward to measure the dephasing rate associated with the inhomogeneous line prepared in a number of different ways. The magnitude of the background can be changed, the height or width of spectral line varied, or a more complex line shape generated.

The CPMG pulse sequence has the possibility of suppressing dephasing arising from perturbations occurring on time scales longer than the separation between the pulses [5]. It should be possible to determine if this is a factor by monitoring the dephasing rates established by a CPMG measurement as a function of the separation between the pulses. Such studies are beyond the scope of this demonstration.

Phenomena investigated need not be restricted to instantaneous spectral diffusion. For example, the sharp spectral feature would be ideal for studying nonoptical Bloch behavior [11]. Likewise, the sharp feature may be of value in the development of quantum computers using rare-earth materials, and indeed it was this aspect that motivated the current work. The technique requires a hole burning material but the hole lifetime need be only 2 orders of magnitude longer than the radiative lifetime, and this condition is met by many materials. Excellent laser stability is also required. However, for most materials the laser stability requirements will be much less severe than the stability already achieved in this work.

In conclusion, we have prepared an isolated and spectrally narrow absorptive feature at the center of the inhomogeneously broadened ${ }^{7} F_{0^{-}}{ }^{5} D_{0}$ transition in $\mathrm{Eu}^{3+}: \mathrm{Y}_{2} \mathrm{SiO}_{5}$. The feature has also a well-defined transition strength. One direct benefit is the creation of a very sensitive probe of ion-ion interactions. In this work we have demonstrated for the first time the use of a multiple pulse sequence to measure the dephasing time of an optical transition. The opportunity now exists to perform more precise manipulations of coherent ensembles using multiple pulse techniques.

[1] C. P. Slichter, Principles of Magnetic Resonance (SpringerVerlag, New York, 1980).

[2] R. R. Ernst, G. Bodenhausen, and A. Wokaun, Principles of Nuclear Magnetic Resonance in One and Two Dimensions (Oxford Science Publications, New York, 1987).

[3] R. M. Macfarlane and R. M. Shelby, in Spectroscopy of Solids Containing Rare Earth Ions, edited by A. A. Kaplyanskii and R. M. Macfarlane (North-Holland, Amsterdam, 1987).

[4] L. E. Erickson, Phys. Rev. A 46, 5899 (1992).

[5] S. Meiboom and D. Gill, Rev. Sci. Instrum. 29, 6881 (1958).

[6] R. W. Equall, Y. Sun, R. L. Cone, and R. M. Macfarlane, Phys. Rev. Lett. 72, 2179 (1994).

[7] R. Yano, M. Mitsunaga, and N. Uesugi, Opt. Lett. 16, 1884 (1991).

[8] T. R. Dyke, M. J. Sellars, G. J. Pryde, N. B. Manson, U. Elman, and S. Kröll, J. Opt. Soc. Am. B 16, 805 (1998).

[9] N. C. Wong, S. S. Kano, and R. G. Brewer, Phys. Rev. A 21, 260 (1980).

[10] R. M. Shelby and R. M. Macfarlane, J. Lumin. 31/32, 839 (1984).

[11] R. G. DeVoe and R. G. Brewer, Phys. Rev. Lett. 50, 1269 (1983). 